01

\title{
Расчет энергии и ширины сверхкритического резонанса в квазимолекуле урана
}

\author{
(ㄱ И.А. Мальцев, В.М. Шабаев, В.А. Зайцев, Р.В. Попов, Ю.С. Кожедуб, Д.А. Тумаков \\ Физический фракультет, Санкт-Петербургский государственный университет, \\ 199034 Санкт-Петербург, Россия \\ e-mail: i.maltsev@sbpu.ru
}

Поступила в редакцию 18.03.2020 г.

В окончательной редакции 18.03.2020 г.

Принята к публикации 25.03.2020 г.

Рассчитаны энергии и ширины сверхкритического резонанса, образующегося при погружении основного состояния квазимолекулы урана в отрицательно-энергетический континуум. Расчеты проведены с учетом полного двухцентрового потенциала ядер. Полученные результаты сравниваются как с соответствующими значениями, полученными в рамках монопольного приближения, так и со значениями, найденными с учетом нескольких низших членов мультипольного разложения двухцентрового потенциала.

Ключевые слова: сверхкритическое поле, распад вакуума.

DOI: $10.21883 /$ OS.2020.08.49703.117-20

\section{Введение}

В сверхсильных кулоновских полях должны наблюдаться качественно новые эффекты квантовой электродинамики, связанные с нестабильностью вакуума [1-6]. Как было впервые показано в работах [2] и [3], при заряде ядра больше критического $Z_{\text {cr }} \geq 173$ нижнее связанное одноэлектронное состояние, погрузившись в отрицательно-энергетический континуум, превращается в резонанс. Если такое состояние было изначально вакантным, то оно может быть заполнено электроном из континуума с вероятностью, зависящей от ширины резонанса. Этот процесс также может быть интерпретирован как распад начального нейтрального вакуума с образованием „заряженного“ вакуума и вылетом позитрона. Ясно, что необходимый критический заряд значительно превышает заряд самых тяжелых из синтезированных ядер. Однако в медленном столкновении двух тяжелых ионов суммарный заряд их ядер вполне может оказаться достаточно большим, чтобы основное состояние образовавшейся квазимолекулы достигло отрицательноэнергетического континуума, и тогда спонтанное рождение пар будет возможным. Наблюдение данного эффекта стало бы прямым подтверждением предсказаний релятивистской квантовой теории в крайне непертурбативном сверхкритическом режиме.

Эксперименты по исследованию сверхкритических столкновений долгое время проводились в GSI (Дармштадт, Германия). Однако никаких следов существования сверхкритического резонанса и распада вакуума обнаружено не было [6-7]. В настоящее время существуют проекты таких исследований на ускорительных комплекcax нового поколения, таких как NICA [8], FAIR [9] и HIAF [10]. Стоит отметить, что, для того чтобы обнаружить следы распада сверхкритического состояния, необходимо выделить спонтанный вклад на фоне динамического процесса рождения электрон-позитронных пар, вызванного зависящим от времени потенциалом сталкивающихся ядер.

В пионерских работах [11-13] процесс рождения электрон-позитронных пар в сверхкритических столкновениях рассматривался в квазистационарном приближении. В рамках этого приближения вероятность рождения пары считалась пропорциональной ширине сверхкритического резонанса в каждой точке траектории сталкивающих ядер. Таким образом, учитывался только спонтанный механизм рождения пар, а динамический механизм игнорировался. При этом ширина резонанса также находилась с использованием различных приближений. Подход, учитывающий динамические эффекты и основанный на численном решении нестационарного уравнения Дирака, впервые был предложен франкфуртской группой $[5,14,15]$. В рамках этого подхода были выполнены расчеты вероятностей рождения электронпозитронных пар в так называемом монопольном приближении, в котором учитывается только сферически симметричный член мультипольного разложения двухцентрового потенциала ядер. Позднее эти результаты были подтверждены в работах [16-20], в том числе и за рамками монопольного приближения.

На основании полученных результатов франкфуртской группой был сделан вывод о невозможности отличить в энергетических спектрах позитронов спонтанный вклад от динамического для упругих столкновений тяжелых ионов. Возможность наблюдать спонтанный распад вакуума была продемонстрирована только для столкновений с гипотетическим „слипанием“ ядер [21,22]. Тем не менее в недавней работе [23] было показано, что свидетельства распада вакуума можно обнаружить в столкновениях тяжелых ионов без какого-либо слипания. 
Предложенный подход основан на различном поведении вероятности рождения пар как функции скорости ионов в докритическом и сверхкритическом случаях. Однако экспериментальное обнаружение распада вакуума согласно предложенному сценарию представляет собой очень сложную задачу.

Для поиска лучших сценариев обнаружения спонтанного механизма рождения пар необходимы расчеты характеристик сверхкритического резонанса. В работах [24-26] для этой цели была использована техника комплексного вращения и комплексного поглощающего потенциала, которые широко применяются в атомной и молекулярной физике для расчета характеристик квазистационарных состояний [27]. При этом в [24] приведены результаты, полученные в монопольном приближении. В работах [25-26] были включены в расчет несколько низших членов мультипольного разложения. Как отмечается в [26], несмотря на то, что авторам в большинстве случаев удалось добиться хорошей сходимости по числу членов мультипольного разложения, при относительно малых и больших межъядерных расстояниях высшие члены могу вносить заметный вклад. Данную проблему может решить только метод, учитывающий полный двухцентровый потенциал.

В представленной работе предложен метод расчета сверхкритического резонанса в полном двухцентровом потенциале. Подход основан на использовании конечного базисного набора вместе с комплексным вращением. Базисные функции строятся на двумерной пространственной сетке, что позволяет учесть полный двухцентровый потенциал, не разлагая его в мультипольный ряд. При этом применяется техника дуального кинетического баланса [28] для аксиально-симметричных систем (А-ДКБ) [29], которая позволяет избежать появления в спектре ложных (шпуриозных) состояний [30]. Метод комплексного вращения заключается в повороте радиальной координаты в комплексную плоскость. В результате такого преобразования волновые функции резонансных состояний становятся квадратично интегрируемыми, что позволяет исследовать их с помощью конечного базиса. Гамильтониан при этом теряет эрмитовость, а соответствующие резонансам энергии принимают комплексные значения. Действительная часть этой энергии соответствует положению резонанса, а мнимая определяет время его жизни.

С помощью разработанного метода проведены расчеты параметров сверхкритического резонанса для квазимолекулы урана $\mathrm{U}_{2}^{183+}$. Расчеты выполнены для различных значений межъядерного расстояния. Полученные результаты сопоставлены с соответствующими монопольными значениями, а также со значениями, полученными в работе [26] с учетом нескольких низших членов мультипольного разложения двухцентрового потенциала.

В настоящей работе полагаем постоянную Планка $\hbar=1$.

\section{Теоретический формализм}

В представленной работе исследуется квазимолекула, состоящая из двух идентичных ядер с зарядом $Z_{1}=Z_{2}=Z_{\text {nucl. }}$ Электронная структура квазимолекулы определяется стационарным уравнением Дирака:

$$
\begin{gathered}
H \psi(\mathbf{r})=E \psi(r), \\
H=c(\boldsymbol{\alpha} \cdot \mathbf{p})+V_{\mathrm{TC}}(\mathbf{r})+\beta m_{e} c^{2} .
\end{gathered}
$$

Здесь $(\boldsymbol{\alpha}, \beta)$ - матрицы Дирака, $m_{e}-$ масса электрона, $c$ - скорость света, $V_{\mathrm{TC}}-$ двухцентровый потенциал ядер:

$$
V_{\mathrm{TC}}(\mathbf{r}, t)=V_{\text {nucl }}(\mathbf{r}+\mathbf{R} / 2)+V_{\text {nucl }}(\mathbf{r}-\mathbf{R} / 2),
$$

где $\mathbf{R}$ - межъядерный вектор, направленный от одного ядра к другому. В данной формуле предполагается, что начало координат находится в центре масс квазимолекулы. Потенциал ядра определяется выражением

$$
V_{\text {nucl }}(\mathbf{r})=\frac{e}{4 \pi} \int d^{3} \mathbf{r}^{\prime} \frac{\rho_{\text {nucl }}\left(\mathbf{r}^{\prime}\right)}{\left|\mathbf{r}-\mathbf{r}^{\prime}\right|} .
$$

Для плотности распределения заряда по ядру $\rho_{\text {nucl }}$ используется модель равномерно заряженного шара.

Введем сферическую систему координат $(r, \theta, \varphi)$ таким образом, что начало координат находится в центре масс, а полярный угол $\theta$ откладывается от оси $z$, которая совпадает с межъядерной осью. Так как гамильтониан симметричен относительно вращений вокруг межъядерной оси, то он коммутирует с оператором соответствующей проекции углового момента $J_{z}$ и решение уравнения (1) можно выбрать в виде

$$
\psi_{m}(r, \theta, \varphi)=\frac{1}{r}\left(\begin{array}{l}
G_{1}(r, \theta) \exp \left[i\left(m-\frac{1}{2}\right) \varphi\right] \\
G_{2}(r, \theta) \exp \left[i\left(m+\frac{1}{2}\right) \varphi\right] \\
i F_{1}(r, \theta) \exp \left[i\left(m-\frac{1}{2}\right) \varphi\right] \\
i F_{2}(r, \theta) \exp \left[i\left(m+\frac{1}{2}\right) \varphi\right]
\end{array}\right),
$$

где $m$ - проекция углового момента на ось $z$, при этом $J_{z} \psi_{m}=m \psi_{m}$. После подстановки выражения (5) в формулу (1) можно отделить координату $\varphi$ и записать уравнение на собственные значения

$$
H_{m}(t) \Phi(r, \theta)=E \Phi(r, \theta)
$$

для функции

$$
\Phi(r, \theta, t)=\left(\begin{array}{c}
G_{1}(r, \theta, t) \\
G_{2}(r, \theta, t) \\
F_{1}(r, \theta, t) \\
F_{2}(r, \theta, t)
\end{array}\right)
$$

Для того чтобы получить параметры сверхкритического резонанса, мы используем метод комплексного 
Энергия $E$ и ширина Г сверхкритического резонанса в квазимолекуле урана $\mathrm{U}_{2}^{183+}$ как функции межъядерного расстояния $R$ (МР). Представлены результаты монопольного приближения (МП), результаты расчетов с полным двухцентровым потенциалом (ДЦ), а также соответствующие значения из работы [26], полученные с учетом низших членов мультипольного разложения потенциала вплоть да значений момента $l_{\max }=4$

\begin{tabular}{c|c|c|c|c|c|c}
\hline MP & \multicolumn{2}{|c|}{ МП } & \multicolumn{2}{c|}{ ДЦ } & \multicolumn{2}{c}{$[26]$} \\
\hline$R, \mathrm{fm}$ & $E, m_{e} c^{2}$ & $\Gamma, \mathrm{keV}$ & $E, m_{e} c^{2}$ & $\Gamma, \mathrm{keV}$ & $E, m_{e} c^{2}$ & $\Gamma, \mathrm{keV}$ \\
\hline 16 & -1.582 & 1.84 & -1.609 & 2.23 & -1.60494 & 2.1471 \\
18 & -1.481 & 1.03 & -1.512 & 1.27 & -1.50877 & 1.2431 \\
20 & -1.391 & 0.48 & -1.425 & 0.66 & -1.42240 & 0.6440 \\
22 & -1.311 & 0.18 & -1.347 & 0.29 & -1.34471 & 0.2859 \\
24 & -1.239 & 0.05 & -1.276 & 0.11 & -1.27462 & 0.1013 \\
26 & -1.173 & 0.01 & -1.213 & 0.03 & -1.21115 & 0.02500 \\
28 & -1.115 & - & -1.155 & - & -1.15342 & $3.3231 \cdot 10^{-3}$ \\
30 & -1.061 & - & -1.102 & - & -1.10073 & $1.2325 \cdot 10^{-4}$
\end{tabular}

вращения, согласно которому радиальная координата преобразуется следующим образом:

$$
r \rightarrow r e^{i \Theta}
$$

где параметр $\Theta$ называется углом комплексного вращения. В результате такого преобразования волновая функция резонанса становится квадратично интегрируемым решением уравнения (6) и ей соответствует комплексное значение

$$
E=E_{0}+i \Gamma / 2
$$

где $E_{0}$ - энергия резонанса, $Г-$ его ширина. Следует отметить, что для сверхкритического резонанса ширина входит в уравнение (9) с положительным знаком, что соответствует вылету позитрона при распаде резонанса.

Уравнение (6) с учетом преобразования (8) решается численно с помощью разложения волновой функции (7) по конечному базисному набору:

$$
\Phi(r, \theta)=\sum_{i=1}^{N} c_{i} u_{i}(r, \theta) .
$$

Здесь $u_{i}(r, \theta)$ - базисные функции, $c_{i}-$ коэффициенты разложения. В данной работе базисные функции строятся из В-сплайнов согласно методу А-ДКБ [29]. В-сплайны, в свою очередь, определены на двумерной сетке в координатах $(r, \theta)$ в сферическом ящике конечного радиуса $L$. Для построения базиса используется $N_{r}$ В-сплайнов, зависящих от радиальной координаты $r$ и $N_{\theta}$ В-сплайнов, зависящих от угловой координаты $\theta$, при этом полное число базисных функций $N=4 N_{r} N_{\theta}$. Подробное описание используемого базисного набора можно найти в работах $[18,20]$.

С помощью разложения (10) и вариационного принципа „повернутое“ согласно формуле (8) уравнение (6) сводится к обобщенной задаче на собственные значения:

$$
\sum_{k=1}^{N} H_{j k}^{(\Theta)} c_{k}^{(\Theta)}=\sum_{k=1}^{N} E^{(\Theta)} S_{j k} c_{k}^{(\Theta)},
$$

где $S_{j k}$ и $H_{j k}^{(\Theta)}$ - элементы матриц перекрывания и гамильтониана соответственно. Следует отметить, что при точном решении преобразованного уравнения Дирака энергия и ширина резонанса не зависят от угла комплексного вращения при его изменении в пределах $\Theta_{\mathrm{c}}<\Theta<\pi / 2$, где $\Theta_{\mathrm{c}}-$ некоторый критический угол. Однако, при использовании конечного базиса точность полученного значения $E^{(\Theta)}$ зависит от $\Theta$. В представленной работе расчеты производились при оптимальном значение $\Theta=\Theta_{\text {opt }}$, которое находилось путем поиска минимума производной $d E^{(\Theta)} / d \Theta$ (подробнее см. в работах $[31,32])$.

\section{Результаты и их обсуждение}

В данной работе проведены расчеты энергии и ширины сверхкритического резонанса в квазимолекуле урана $\mathrm{U}_{2}^{183+}$ для различных значений межъядерного расстояния $R$. Квазимолекула становится сверхкритической (т.е. происходит погружение основного состояния в отрицательно-энергетический континуум) при $R<R_{\mathrm{c}}$, где критическое расстояние $R_{\mathrm{c}} \approx 34.7 \mathrm{fm}$. При межъядерном расстоянии $R_{0} \approx 16 \mathrm{fm}$ ядра почти „касаются“ друга друга поверхностями. Таким образом, имеет смысл исследовать сверхкритический резонанс в диапазоне межъядерных расстояний $R_{0} \leq R<R_{\mathrm{c}}$.

В настоящей работе мы использовали модель равномерно заряженного шара для распределения заряда по ядру. При этом использовалось значение радиуса ядра $R_{\text {nucl }}=\sqrt{5 / 3} R_{\mathrm{rms}}$ с $R_{\mathrm{rms}}=5.8569 \mathrm{fm}$ [33]. Отметим, что параметры сверхкритического резонанса достаточно чувствительны к модели и радиусу ядра. 
Для построения базиса использовалось $N_{r}=200$ В-сплайнов по $r$-координате и $N_{\theta}=15$ В-сплайнов по $\theta$-координате. При этом применялась равномерная сетка по угловой координате, радиальная сетка при $r<R$ была равномерная с шагом $1 \mathrm{fm}$, далее узлы сетки экспоненциально разбегались вплоть до границы ящика $L=10^{5} \mathrm{fm}$.

Полученные результаты представлены в таблице. Там же приведены значения, полученные в рамках монопольного приближения и соответствующие величины из работы [26]. В монопольном приближении учитывается только первый член мультипольного разложения, соответствующий моменту $l_{\max }=0$. В работе [26] были учтены еще несколько членов этого разложения вплоть до $l_{\max }=4$. Для межъядерного расстояния $R>26 \mathrm{fm}$ ширина резонанса столь мала, что нашим методом не удалось получить для нее достаточно стабильное значение. Как видно из таблицы, наши значения для энергии хорошо согласуются со значениями из работы [26], при этом они всегда лежат ниже. Полученные значения для ширины резонанса отличаются сильнее. В целом можно сделать вывод о том, что при более точном учете двухцентрового потенциала глубина погружения уровня и его ширина увеличиваются, при этом относительное изменение ширины гораздо больше. Представляется довольно неожиданным то, что ширина достаточно сильно увеличивается при более точном учете потенциала даже для $R=16 \mathrm{fm}$, хотя считается, что при малом межъядерном расстоянии монопольное приближение должно работать очень хорошо.

\section{Заключение}

Выполнены расчеты энергии и ширины сверхкритического резонанса для квазимолекулы урана $\mathrm{U}_{2}^{183+}$. Результаты получены для различных межьядерных расстояний. Для расчетов использовался метод конечного базисного набора в сочетании с комплексным вращением. Подобные расчеты с учетом полного двухцентрового потенциала проведены впервые и могут рассматриваться как самые точные на сегодняшний день.

\section{Финансирование работы}

Работа выполнена при финансовой поддержке РФФИ в рамках проекта № 18-32-00723.

\section{Конфликт интересов}

Авторы заявляют, что у них нет конфликта интересов.

\section{Список литературы}

[1] Pomeranchuk I., Smorodinsky J. // J. Phys. USSR. 1945. V. 9. P. 97.

[2] Gershtein S.S., Zeldovich Y.B. // Zh. Eksp. Teor. Fiz. 1969. V. 57. P. 654; Sov. Phys. JETP. 1970. V. 30. P. 358.

[3] Pieper W., Greiner W. // Z. Phys. 1969. V. 218. P. 327.

[4] Zeldovich Y.B., Popov V.S. // Sov. Phys. Usp. 1972. V. 14. P. 673.

[5] Greiner W., Müller B., Rafelski J. // Quantum Electrodynamics of Strong Fields Berlin: Springer-Verlag, 1985.

[6] Müller-Nehler U., Soff G. // Phys. Rep. 1994. V. 246. P. 101.

[7] Ahmad I. et al. // Phys. Rev. C. 1999. V. 60. P. 064601.

[8] Ter-Akopian G.M., Greiner W., Meshkov T.N., Oganessian Y.T., Reinhardt J., Trubnikov G.V. // Int. J. Mod. Phys. E. 2015. V. 24. P. 1550016.

[9] Gumberidze A., Stöhlker Th., Beyer H.F., Bosch F., BräuningDemian A., Hagmann S., Kozhuharov C., Kühl Th., Mann R., Indelicato P., Quint W., Schuch R., Warczak A. // Nucl. Instrum. Methods Phys. Res. B. 2009. V. 267. P. 248.

[10] Ma X., Wen W.Q., Zhang S.F., Yu D.Y., Cheng R., Yang J., Huang Z.K., Wang H.B., Zhu Z.L., Cai X., Zhao Y.T., Mao L.J., Yang J.C., Zhou X.H., Xu H.S., Yuan Y.J., Xia J.W., Zhao H.W., Xiao G.Q., Zhan W.L. // Nucl. Instrum. Methods Phys. Res., Sect. B. 2017. V. 408. P. 169.

[11] Gershtein S.S., Popov V.S. // Lett. Nuovo Cimento. 1973 V. 6. P. 593.

[12] Popov V.S. // Zh. Eksp. Teor. Fiz. 1973. V. 65. P. 35.; Sov. Phys. JETP. 1974. V. 38. P. 18.

[13] Peitz H., Müller B., Rafelski J., Greiner W. // Lett. Nuovo Cimento. 1973. V. 8. P. 37.

[14] Reinhardt J., Müller B., Greiner W. // Phys. Rev. A. 1981. V. 24. P. 103.

[15] Müller U., de Reus T., Reinhardt J., Müller B., Greiner W., Soff G. // Phys. Rev. A. 1988. V. 37. P. 1449.

[16] Maltsev I.A., Shabaev V.M., Tupitsyn I.I., Bondarev A.I., Kozhedub Y.S., Plunien G., Stöhlker Th. // Phys. Rev. A. 2015. V. 91. P. 032708 .

[17] Bondarev A.I, Tupitsyn I.I., Maltsev I.A., Kozhedub Y.S., Plunien G. // Eur. Phys. J. D. 2015. V. 69. P. 110.

[18] Maltsev I.A., Shabaev V.M., Tupitsyn I.I., Kozhedub Y.S., Plunien G., Stöhlker Th. // Nucl. Instrum. Methods Phys. Res. B. 2017. V. 408. P. 97.

[19] Popov R.V., Bondarev A.I., Kozhedub Y.S., Maltsev I.A., Shabaev V.M., Tupitsyn I.I, Ma X., Plunien G., Stöhlker Th. // Eur. Phys. J. D. 2018. V. 72 P. 115.

[20] Maltsev I.A., Shabaev V.M., Popov R.V., Kozhedub Y.S., Plunien G., Ma X., Stöhlker Th. // Phys. Rev. A. 2018. V. 98. P. 062709.

[21] Reinhardt J., Greiner W. // Supercritical fields and the decay of the vacuum, in Proceeding of the Memorial Symposium for Gerhard Soff. EP Systema, Budapest, 2005. P. 181-192.

[22] Rafelski J., Kirsch J., Müller B., Reinhardt J., Greiner W. // New Horizons in Fundamental Physics. Editors: Schramm S., Schäfer M. 2016. Springer, P. 211-251. arXiv: 1604.08690.

[23] Maltsev I.A., Shabaev V.M., Popov R.V., Kozhedub Y.S., Plunien G., Ma X., Stöhlker Th., Tumakov D.A. // Phys. Rev. Lett. 2019. V. 123. P. 113401.

[24] Ackad E., Horbatsch M. // Phys. Rev. A. 2007. V. 75. P. 022508.

[25] Ackad E., Horbatsch M. // Phys. Rev. A. 2007. V. 76. P. 022503. 
[26] Marsman A., Horbatsch M. // Phys. Rev. A. 2011. V. 84. P. 032517.

[27] Moiseyev N. // Phys. Rep. 1998. V. 302. P. 211.

[28] Shabaev V.M., Tupitsyn I.I., Yerokhin V.A., Plunien G., Soff G. // Phys. Rev. Lett. 2004. V. 93. P. 130405.

[29] Rozenbaum E.B., Glazov D.A., Shabaev V.M., Sosnova K.E., Telnov D.A. // Phys. Rev. A. 2014. V. 89. P. 012514.

[30] Тупицын И.И., Шабаев В.М. // Опт. и спектр. 2008. Т. 105, C. 203; Tupitsyn I.I., Shabaev V.M. // Opt. Spektrosc. 2008. V. 105 P. 203.

[31] Zaytsev V.A., Maltsev I.A., Tupitsyn I.I., Shabaev V.M. // Phys. Rev. A. 2019. V. 100. P. 052504.

[32] Зайцев В.А., Мальцев И.А., Тупицын И.И., Шабаев В.М., Иванов В.Ю. // Опт. и спектр. 2020. Т. 128. С. 318.

[33] Kozhedub Y.S, Andreev O.V., Shabaev V.M., Tupitsyn I.I., Brandau C., Kozhuharov C., Plunien G., Stöhlker Th. // Phys. Rev. A. 2008. V. 77. P. 032501. 